\title{
The regulation and function of microRNA-377/RASSF8 signaling axis in gastric cancer
}

\author{
XIAOBO BO*, YUSHENG CHEN* ${ }^{*}$, WEIZHONG SHENG*, \\ YUDA GONG, HAIYU WANG, WEIDONG GAO and BO ZHANG
}

Department of General Surgery, Zhongshan Hospital, Fudan University, Shanghai 200032, P.R. China

Received March 23, 2015; Accepted June 15, 2017

DOI: $10.3892 / \mathrm{ol} .2018 .7740$

\begin{abstract}
Gastric cancer is a major cause of cancer-associated mortality worldwide. The aberrant expression of microRNA (miRNA) is involved in tumorigenesis. Ras proteins transfer information from the extracellular environment to internal cellular compartments and are essential in numerous signal transduction pathways. To investigate the regulation, function and clinical significance of the miRNA377/Ras association domain family (RASSF) 8 signaling axis in gastric cancer, reverse transcription-quantitative polymerase chain reaction, immunohistochemistry, cell counting kit-8, western blotting, and Transwell assays were used. The results revealed that expression of RASSF8 was significantly upregulated in normal gastric tissues compared with gastric cancer, which was further confirmed by immunohistochemical analysis, and its expression level was increased in normal gastric cells compared with gastric cancer cell lines. However, the expression of miR-377 was significantly upregulated in gastric cancer compared with normal gastric tissues. In addition, RASSF8 overexpression in BGC-823 gastric cancer cells significantly inhibited the proliferation, apoptosis and invasive abilities of cells. Whereas miR-377 attenuated these effects due to downregulated RASSF8 expression by directly targeting its 3'-untranslated region. Furthermore, in the current study, miR-377 was not able to reverse the effects of RASSF8 overexpression on gastric cancer cells. Collectively, the RASSF8 gene may represent a novel molecular target involved in gastric cancer development and may be useful in targeted therapy of patients with gastric cancer.
\end{abstract}

Correspondence to: Dr Bo Zhang or Dr Weidong Gao, Department of General Surgery, Zhongshan Hospital, Fudan University, 180 Fenglin Road, Shanghai 200032, P.R. China

E-mail: zhang.bo@zs-hospital.sh.cn

E-mail: gao.weidong@zs-hospital.sh.cn

*Contributed equally

Key words: gastric cancer, microRNA-377, Ras association domain family member 8 , Ras association domain family member 8 overexpression lentivirus, molecular target

\section{Introduction}

Gastric cancer is the fourth most commonly diagnosed cancer type and is the second leading cause of cancer-associated mortality worldwide (1). Numerous molecules are responsible for gastric cancer tumorigenesis, and the cause of carcinogenesis is complicated involving the dysregulation of oncogenes and tumor suppressors (2). Gastrectomy remains the standard treatment for gastric cancer clinically (3). The overall 5 -year relative survival rate for gastric cancer is $\sim 28 \%$ and none of the current treatment modalities are able to influence the overall survival rates (4). The recurrence rate remains high due to the absence of specific symptoms, thus rendering early diagnosis of this fatal disease difficult (4), and the prognosis for patients with the advanced stage remains poor. The outcome of patients with gastric cancer is determined primarily by the presence or absence of metastasis (5). Therefore, developing novel prognostic factors and therapeutic strategies is essential, and identification of the precise molecular mechanisms that modulate malignant transformation is necessary.

As previously reported, Ras genes frequently undergo activating mutations in several human and animal cancer types (6-8). However, the expression of Ras genes in gastric cancer and its clinical values remain to be elucidated. Ras proteins are essential in numerous signal transduction pathways that transfer information from the extracellular environment to internal cellular compartments (9). Thus, mutation-induced deregulation of the Ras superfamily, and upstream and downstream signaling components result in important alterations to healthy cell growth control and differentiation, in addition, they serve an important role in mechanisms of tumor formation $(8,10)$. In mammals, the Ras signal transduction pathway is mediated by several effectors, including proteins with a Ras-associated (RalGDS/AF-6) (RA)-domain, and the RA-domain is a common feature of genes in the Ras association domain family (RASSF) $(9,11)$. However, the importance of RASSF protein functions remains to be clarified and the Ras-binding status of RASSF family members, particularly RASSF8, remain unknown (12).

The complex interaction among different etiological factors leads to genetic and epigenetic alterations of proto-oncogenes, and tumor suppressor genes, which underlie the pathogenesis of cancer. It has long been considered that dysregulation of these genes results in abnormal function or expression of oncogenic 
and tumor suppressor proteins (2). MicroRNAs (miRNAs) are small non-coding RNA molecules that are generated within cells, which serve a role in post-transcriptional gene regulation and in almost any cellular biological function. Aberrant expression of miRNAs has been identified in cancerous transformation and progression (13). miRNA has been demonstrated to be involved in the pathogenesis of gastric cancer (14) and numerous other types of cancer $(15-17,19)$. Furthermore, miRNA represses the expression of its target genes through base pairing with endogenous mRNAs to perform its biological functions, and miRNA genes have been characterized as novel proto-oncogenes or tumor suppressor genes in gastric carcinogenesis (20).

In the present study, the clinical significance of microRNA-377/RASSF8 signaling axis was evaluated, in addition, the expression and function of miRNA377/RASSF8 proteins were analyzed in vitro. The correlation between miRNA377 and RASSF8 protein expression, and clinicopathological variables in cases of gastric cancer were also investigated.

\section{Materials and methods}

Patients and samples. The present study was approved by the Medical Ethics Committee of Shanghai Zhongshan Hospital, Fudan University (Shanghai, China). Prior written informed consent was obtained from each patient. A total of 10 patients with gastric cancer ( 6 male, 4 female; age range, 39-58 years) were included in the present study. The specimens were handled and made anonymous according to the ethical and legal standards. Paired tissue specimens (tumor and adjacent normal mucosa) from 10 patients with gastric cancer were obtained and histologically confirmed by a pathologist at Shanghai Zhongshan Hospital. The samples were derived from patients who had not received adjuvant treatment, including radiotherapy or chemotherapy prior to surgery in order to eliminate potential treatment-induced changes to gene expression profiles. Following excision, tissue samples were immediately snap frozen in liquid nitrogen and stored at $-80^{\circ} \mathrm{C}$ until RNA extraction.

Immunohistochemistry and assessment of RASSF8 expression. Immunohistochemical staining was performed for RASSF8 to evaluate immunoreactivity in the aforementioned tissue samples (21) using the Elivision ${ }^{\text {TM }}$ Plus Two-Step system (Fuzhou Maixin Biotech Co., Ltd., Fuzhou, China). Paraffin-embedded tissue sections (5- $\mu \mathrm{m}$ thick) were prepared. Slides were deparaffinized, rehydrated and subjected to microwave heat antigen retrieval in $0.01 \mathrm{M}$ citrate buffer $(\mathrm{pH}$ 6.0) for $20-25 \mathrm{~min}$ at $25^{\circ} \mathrm{C}$. Following blocking of endogenous peroxidase activity with $3 \% \mathrm{H}_{2} \mathrm{O}_{2}$ for $15 \mathrm{~min}$ at $25^{\circ} \mathrm{C}$, the sections were incubated with a primary antibody directed against RASSF8 (dilution, 1:300; cat. no. ab214324; Abcam, Cambridge, MA, USA) overnight at $4^{\circ} \mathrm{C}$. Following washing with PBS for 3 times $\left(10 \mathrm{~min}\right.$ each, $\left.25^{\circ} \mathrm{C}\right)$, the specimens were incubated with $\mathrm{F}\left(\mathrm{ab}^{\prime}\right) 2$-goat anti-rabbit immunoglobulin $\mathrm{G}$ (IgG) $(\mathrm{H}+\mathrm{L})$ secondary antibody (dilution, 1:200; cat. no. Q-11401MP; Thermo Fisher Scientific Inc., Waltham, MA, USA) at $37^{\circ} \mathrm{C}$ for $1 \mathrm{~h}$ and subsequently washed with PBS three times. Immunoreactivity was visualized using the chromogen, 3,3'diaminobenzidine (Fuzhou Maixin Biotech Co., Ltd.). Slides were then counterstained with $0.1 \%$ hematoxylin solution for $5 \mathrm{~min}$ at $25^{\circ} \mathrm{C}$.

The immunostaining frequency for each tumor was scored as follows: 0 (<10\%); 1 (10-30\%); 2 (31-60\%); and $3(>61 \%)$. Staining intensity was documented as: 0 , no immunostaining; 1 , weak; 2 , moderate and 3 , strong. Total immunostaining score results were calculated from the multiplication of both parameters. Samples were scored totally as follows: A scale of 0 (-, total immunostaining score, 0$)$; 1+ (score range, 1-2); $2+(++$, total immunostaining score, $3 \sim 4)$; and $3+(+++$, total immunostaining score, $6 \sim 7)$. Immunostaining was assessed by an experienced pathologist who was blinded to the clinical data of the patients. RASSF8 expression was determined by assessing the percentage and intensity of stained tumor cells. For RASSF8 protein, immunostainings were scored as strong $(2++$ and $3+++)$, weak or negative $(1+$ and 0$)$ according to the rate of labeled tumor cells and the membrane staining intensity.

Cell culture. The human AGS, BGC-823, HGC-27, MKN-45 and SGC-7901 gastric cancer cell lines, GES-1 normal gastric cell line, and HEK 293T cells were purchased from the Cell Bank of Chinese Academy of Science (Shanghai, China). AGS, BGC-823, MKN-45, SGC-7901 and HEK 293T cells were cultured in Dulbecco's modified Eagle's medium (DMEM). HGC-27 and GES-1 were cultured in RPMI-1640. In each case, the medium was supplemented with $10 \%$ fetal bovine serum (FBS) all from Thermo Fisher Scientific, Inc. (Gibco) and $1 \%$ penicillin and streptomycin (Sigma-Aldrich; Merck $\mathrm{KGaA}$, Darmstadt, Germany). Cells were maintained at $37^{\circ} \mathrm{C}$ in a humidified atmosphere consisting of $5 \% \mathrm{CO}_{2}$.

RNA extraction and reverse transcription-quantitative polymerase chain reaction $(R T-q P C R)$. Total RNA was extracted from cell lines or frozen tissues using TRIzol reagent and miRNeasy mini kit (Invitrogen; Thermo Fisher Scientific, Inc.). The RT-qPCR was performed using All-in-One ${ }^{\mathrm{TM}}$ miRNA qRT-PCR Detection kit (GeneCopoeia Inc., Rockville, MD, USA) according to the manufacturer's protocol. The iQ-5 system (Bio-Rad Laboratories, Inc., Hercules, CA, USA) was used to monitor the PCR in real-time. The PCR cycling profile was as follows: Denaturation at $95^{\circ} \mathrm{C}$ for $2 \mathrm{~min}$; followed by 40 cycles of annealing at $95^{\circ} \mathrm{C}$ for $5 \mathrm{sec}$; and extension at $60^{\circ} \mathrm{C}$ for $35 \mathrm{sec}$. The average cycle threshold $(\mathrm{Cq})$, from triplicate assays, was used for further calculations. Relative expression levels were normalized to the control (GES-1, control cell line; normal gastric mucosal tissue, control tissue). The endogenous U6 snRNA was chosen as the internal control. The $2^{-\Delta \Delta \mathrm{Cq}}$ method (22) was used to quantify the relative amount of miR-377/RASSF8, where $\Delta \Delta \mathrm{Cq}=[(\mathrm{CqmiR}-377 / \mathrm{RASSF})-\mathrm{CqU} 6 \mathrm{RNA}]-(\mathrm{Cq}$ control-CqU 6RNA).

Lentivirus and lentivirus infection. RASSF8 overexpression lentivirus was purchased from Shanghai R\&S Biotechnology Co., Ltd. (Shanghai, China). BGC-823 cells were seeded into $3.5-\mathrm{cm}$ dishes $\left(1 \times 10^{6}\right.$ cells/dish) $24 \mathrm{~h}$ prior to lentivirus infection. The next day, lentivirus was added into dishes with a multiplicity of infection of 4 to infect cells. The infection efficiency was detected using fluorescence microscopy analysis of 
Table I. RASSF8 expression detected by immunohistochemistry in gastric tissues.

\begin{tabular}{|c|c|c|c|c|c|}
\hline \multirow[b]{2}{*}{ Histological type } & \multirow[b]{2}{*}{ Number of patients } & \multicolumn{4}{|c|}{ RASSF8 expression, $\mathrm{n}(\%)$} \\
\hline & & - & + & ++ & +++ \\
\hline Normal gastric mucosa & 10 & $0(0)$ & $0(0)$ & $1(10)$ & $9(90)$ \\
\hline Gastric cancer & 10 & $0(0)$ & $8(80)$ & $2(20)$ & $0(0)$ \\
\hline
\end{tabular}

$\mathrm{P}<0.05$ overall difference in RASSF8 expression, comparison between adjacent non-cancerous mucosa (normal gastric mucosa) and gastric cancer tissues. RASSF8, Ras association domain family 8.

A

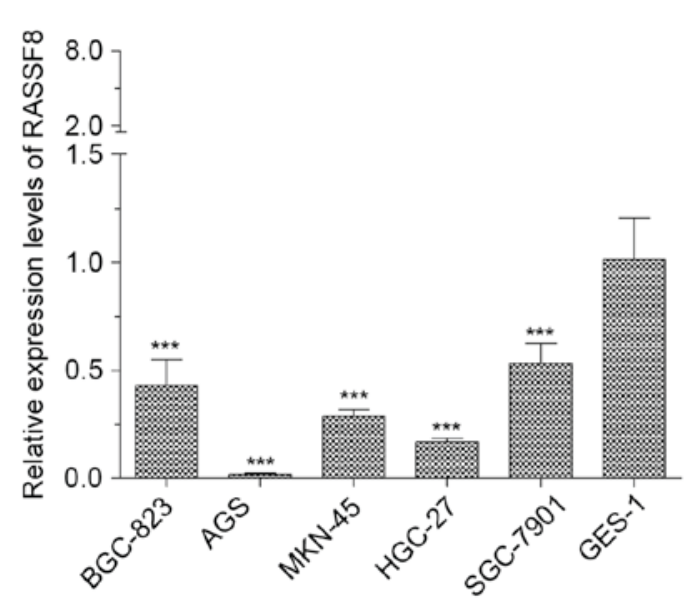

C

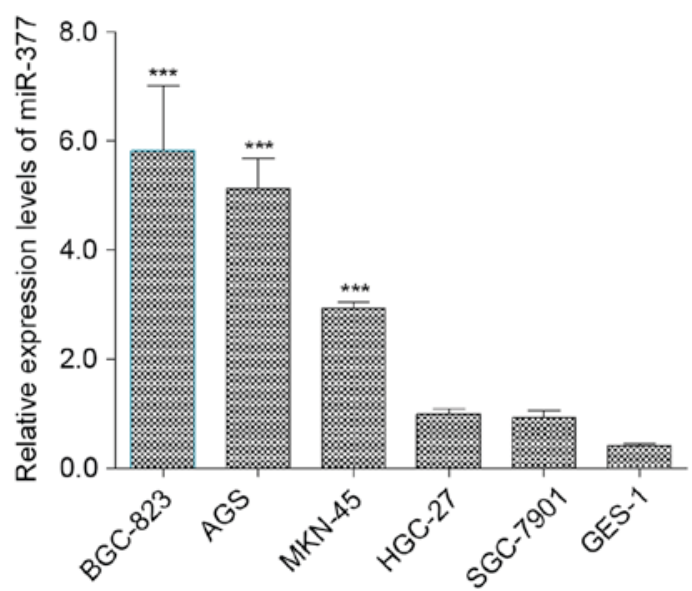

B

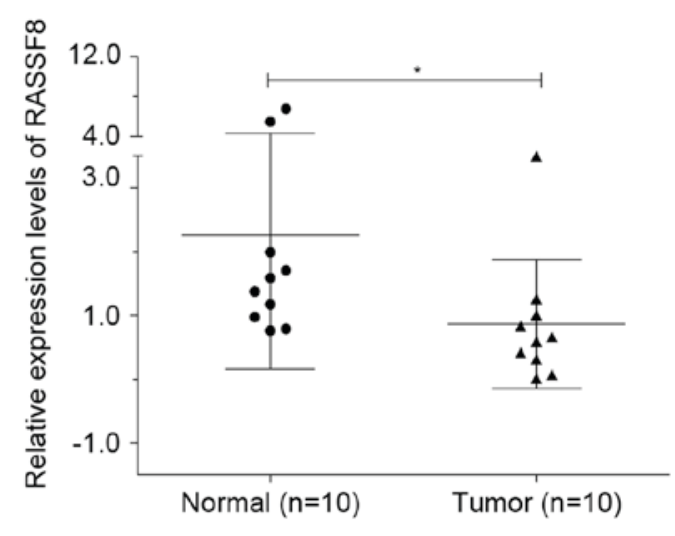

D

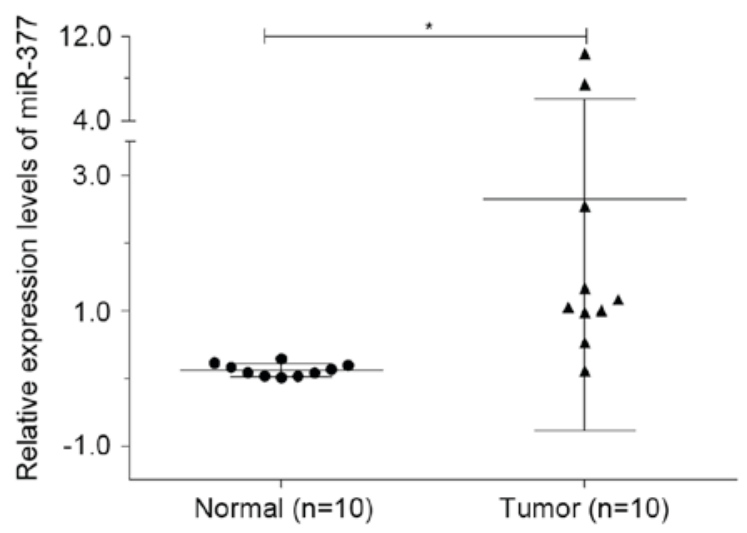

Figure 1. Expression of RASSF8 and miR-377 in cell lines, gastric cancer tissues and adjacent normal tissues. (A) Levels of RASSF8 in the human GES-1 normal cells were significantly higher compared with that in BGC-823, AGS, MKN-45, HGC-27 and SGC-7901 (*** P<0.001). (B) A significant decrease in the RASSF8 levels was identified in gastric cancer tissues as compared with that of adjacent normal tissues ( $\mathrm{n}=10$; $\left.{ }^{*}<<0.05\right)$. (C) Levels of miR-377 in BGC-823, AGS, MKN-45 were significantly higher compared with the levels of miR-377 in GES-1 (**** $\mathrm{P}<0.001)$. No significant difference was identified in the miR-377 levels of HGC-27, SGC-7901 and GES-1 cells. (D) A significant increase in the miR-377 level was identified in gastric cancer tissues as compared with that of adjacent normal tissues $\left(\mathrm{n}=10\right.$; $\left.{ }^{*} \mathrm{P}<0.05\right)$. Data are presented as the mean \pm standard deviation of three independent experiments. RASSF8, Ras association domain family $8 ;$ miR, microRNA.

green fluorescence protein $48 \mathrm{~h}$ after infection, and was only accepted when the efficiency was $>90 \%$.

miRNA mimics, plasmids and transfection. The miR-377 mimic and negative control (miR-NC) were purchased from Shanghai GenePharma, Co., Ltd. (Shanghai, China). The transfection of miRNAs $(50 \mathrm{nM})$ was performed using X-tremeGENE (Roche Applied Science, Rotkreuz, Switzerland) according to the manufacturer's protocol. The 3'-untranslated region (UTR) was PCR-amplified from BGC-823 genomic DNA and cloned downstream of the luciferase gene in the pGL vector (Promega Corporation, Madison, WI, USA). 

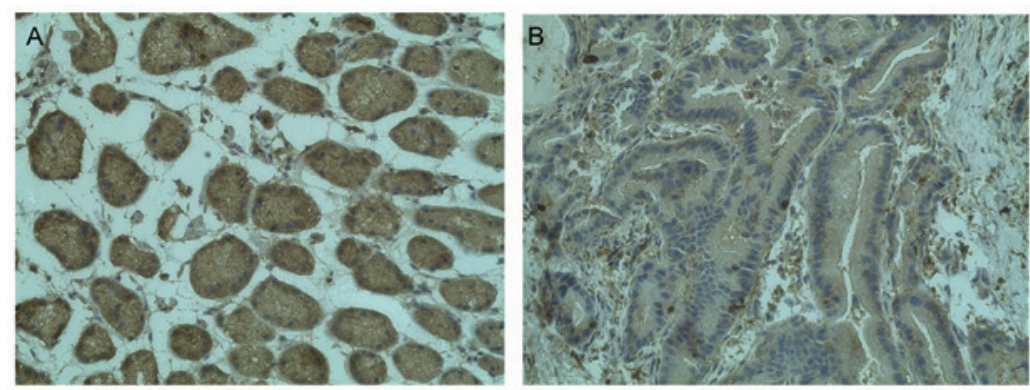

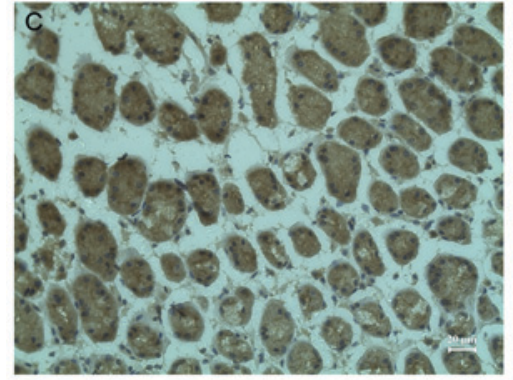

Adjacent tissue

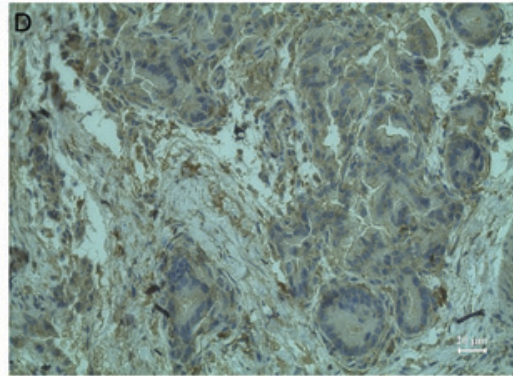

Gastric cancer tissue

Magnification $\times 400$

Figure 2. RASSF8 protein expression in normal gastric mucosa and gastric cancer tissue was detected by immunohistochemistry (magnification, $\mathrm{x} 400$; scale bar, $20 \mu \mathrm{m})$. (A and C) Normal gastric mucosa, RASSF8 (+++); (B and D) gastric cancer tissue, RASSF8 (+). RASSF8, Ras association domain family 8.

Western blot analysis. Total protein from cells was extracted according to the protocol of the Whole Protein Extraction kit (KGP2100; Nanjing KeyGen Biotech Co., Ltd., Nanjing, China). Equivalent quantities (40 $\mu \mathrm{g}$ protein/lane) of protein were separated by $10 \%$ SDS-PAGE and transferred to polyvinylidene difluoride membranes. Membranes were blocked with $10 \%$ skimmed milk (10\% bovine serum albumin (KGY00810, Nanjing KeyGen Biotech Co.,Ltd) for phosphorylated-protein), and then incubated with the appropriate primary antibody overnight at $4^{\circ} \mathrm{C}$. Membranes were subsequently washed with Tris-Buffered saline-Tween 20 and incubated with the corresponding horseradish peroxidase-conjugated AffiniPure goat anti-rabbit $\operatorname{IgG}(\mathrm{H}+\mathrm{L})$ secondary antibody (dilution, 1:200; cat. no. 111-035-003; Jackson ImmunoResearch Inc., West Grove, PA, USA) at $25^{\circ} \mathrm{C}$ for $1 \mathrm{~h}$. Bound secondary antibody was visualized using an enhanced chemiluminescence system (Pierce Biotechnology, Rockford, IL, USA). The primary antibodies used were as follows: Anti-GAPDH (1:1,000; cat. no. 2118; Cell Signal Technology, Inc., Danvers, MA, USA); and anti-RASSF8 (1:2,000; cat. no. ab126110; Abcam). The results were normalized to GAPDH to correct for loading.

Cell viability assay. A total of $1 \times 10^{4}$ BGC- 823 cells/well were plated in 96-well plates and cultured for 1-6 days in normal conditions. Cell viability was assessed using Cell Counting kit-8 (Dojindo, Kumamoto, Japan). A total of $10 \mu \mathrm{l}$ of cell counting assay kit-8 (CCK-8) solution was added daily to each well. After treatment with $\mathrm{CCK}-8$ at $37^{\circ} \mathrm{C}$ for $2 \mathrm{~h}$, the absorbance at $450 \mathrm{~nm}$ was measured using a microplate reader.

Colony formation assay. The transfected cells were plated in 6 -well plates at a density of $1 \times 10^{3}$ cells/well and incubated in culture medium (DMEM containing 10\% FBS) for 10 days without any disturbance. The cells were fixed with $70 \%(\mathrm{v} / \mathrm{v})$ ethanol for $15 \mathrm{~min}$ at $25^{\circ} \mathrm{C}$ and stained with $0.5 \%$ crystal violet for $1 \mathrm{~h}$ at $37^{\circ} \mathrm{C}$. Visible colonies were counted in 10 different fields and the mean value was calculated.

Cell cycle assay. The cells were seeded into 6-well plates at a density of $1 \times 10^{5}$ cells/well after transfection for $24 \mathrm{~h}$, and maintained in DMEM/RPMI-1640 containing 10\% FBS. After being cultured for $24,4 \mathrm{~h}$ and $72 \mathrm{~h}$, the cells were harvested and fixed in $70 \%$ (v/v) ice-cold ethanol for $15 \mathrm{~min}$ at $4^{\circ} \mathrm{C}$, then stored at $-20^{\circ} \mathrm{C}$ overnight. The fixed cells were washed twice with PBS $(1 \mathrm{ml})$ and stained with propidium iodide (PI) solution (Invitrogen; Thermo Fisher Scientific, Inc.) $(10 \mu \mathrm{g} / \mathrm{ml})$ including RNase $(0.5 \mathrm{mg} / \mathrm{ml})$ for $30 \mathrm{~min}$ at $4^{\circ} \mathrm{C}$ in the dark. Flow cytometry was then performed using a FACSCalibur cytometer (BD Biosciences, Franklin Lakes, NJ, USA) to examine the cell cycle distribution. Experiments were performed in triplicate.

Apoptosis assay. A total of $2 \times 10^{5}$ lentivirus-infectedcells BGC-823 cells/well were plated in 6-well plates, and then transfected with miR-NC and miR-377 mimics for $48 \mathrm{~h}$. The transfected cells were harvested by brief trypsinization and centrifugation $(170 \mathrm{xg})$ for $5 \mathrm{~min}$ at $4{ }^{\circ} \mathrm{C}$, washed in ice-cold PBS and resuspended in PBS containing Annexin V-fluorescein isothiocyanate. PI (Thermo Fisher Scientific, Inc.) was then added, and the cells were incubated for $30 \mathrm{~min}$ at $20^{\circ} \mathrm{C}$. A total of $1 \times 10^{4}$ cells/sample were acquired using a FACScan flow cytometer and the proportions of labeled cells were analyzed using Paint-A-Gate software (BD Paint-A-Gate Pro for Windows; version 649728; BD Biosciences).

Invasion assay in vitro. The upper surface of a polycarbonate membrane in a Transwell filter insert was coated with Matrigel $(10 \mu \mathrm{g} / \mu \mathrm{l} ; 30 \mu \mathrm{l})$. After $30 \mathrm{~min}$ of incubation 
A

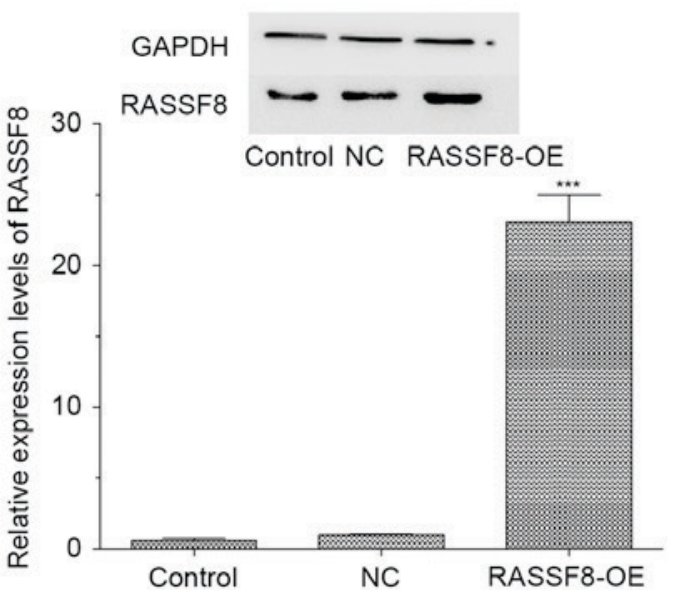

B

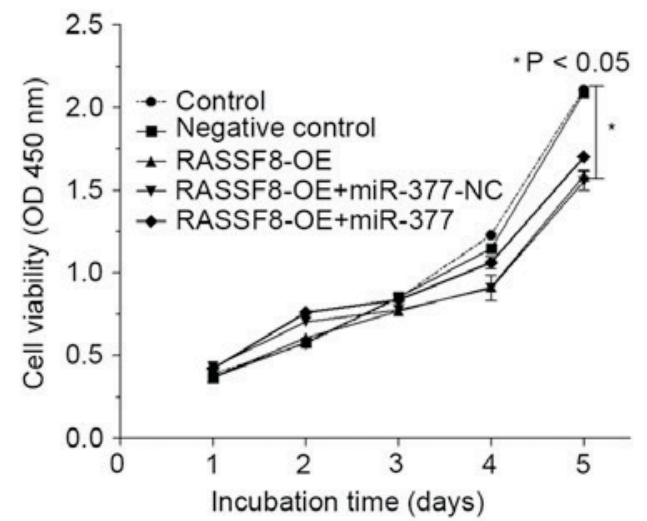

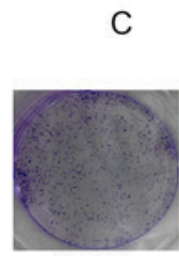

Control

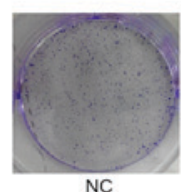

NC
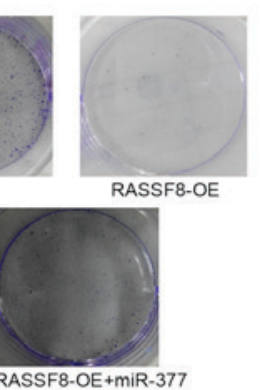

RASSF8-OE+miR-377-NC RASSF8-OE+miR-377

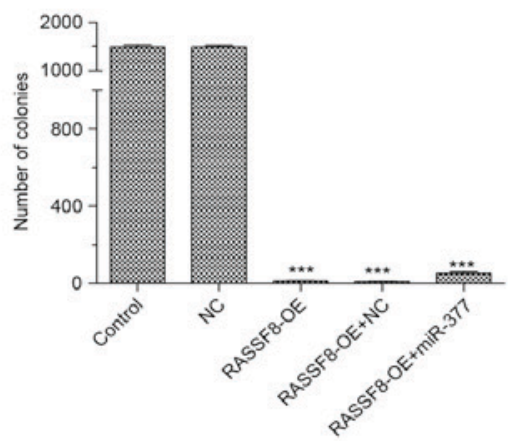

D

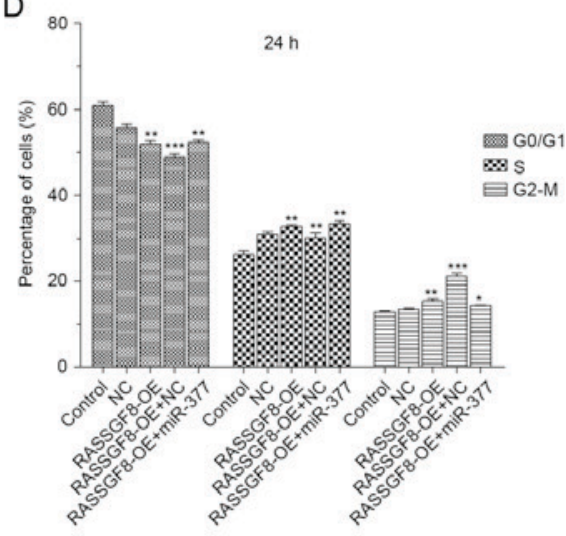

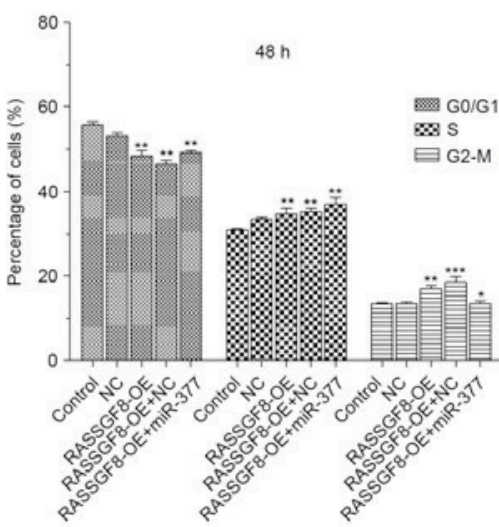

$72 \mathrm{~h}$

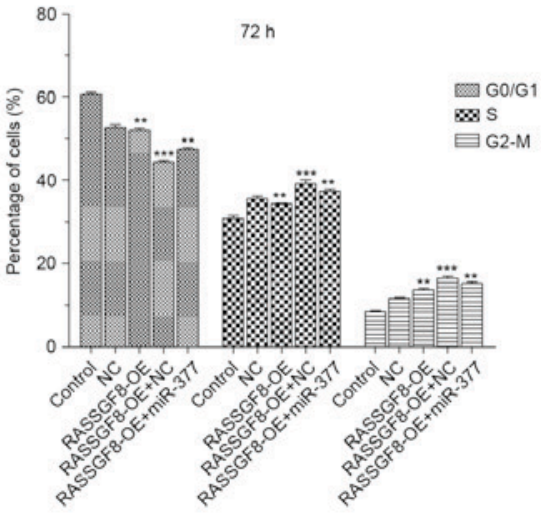

Figure 3. RASSF8 inhibits cell growth in BGC-823 cells. (A) Western blot analyses of RASSF8 levels in control, NC and RASSF8-OE transfected BGC-823 cells. (B) Effects of RASSF8 and miR-377 on tumor cell proliferation at 0, 1,2,3,4 and 5 day using the CCK8 assay. (C) Representative quantification of colony formation in BGC-827 cells transfected with control, negative control, RASSF8-OE and RASSF8 overexpression with miR-377 (RASSF8-OE+ miR-377). (D) Cell cycle profiles of BGC-823 cells transfected with control, negative control, RASSF8-OE and RASSF8-OE+ miR-377. Data are presented as the mean \pm standard deviation of three independent experiments. ${ }^{*} \mathrm{P}<0.05,{ }^{* *} \mathrm{P}<0.01$ and ${ }^{* * *} \mathrm{P}<0.001$ vs. control. RASSF8, Ras association domain family 8 ; miR, microRNA; OE, overexpression; NC, negative control.

at $37^{\circ} \mathrm{C}$, the Matrigel solidified and served as the extracellular matrix for tumor cell invasion analyses. The cells were harvested in $100 \mu 1$ serum-free growth medium and seeded into the upper compartment of the chamber. BGC-823 cells or lentivirus-infected cells were seeded into the upper chambers at the density of $8 \times 10^{5}$ cells in $100 \mu 1$ serum-free DMEM. The lower chambers were filled with $500 \mu \mathrm{l}$ RPMI-1640 containing $10 \%$ FBS. After $24 \mathrm{~h}$ of incubation, non-invading cells on the top of the membrane were removed by scraping. Invaded cells on the bottom of the membrane were fixed with $4 \%$ paraformaldehyde for $15 \mathrm{~min}$ at $25^{\circ} \mathrm{C}$, followed by staining with $0.5 \%$ crystal violet for $20 \mathrm{~min}$ at $25^{\circ} \mathrm{C}$. The invaded cells on the membrane were then counted in three random microscopic fields/well, using ImageJ software (k 1.45; National Institutes of Health, Bethesda, MD, USA) (23). Invasion studies were repeated three times. 
A

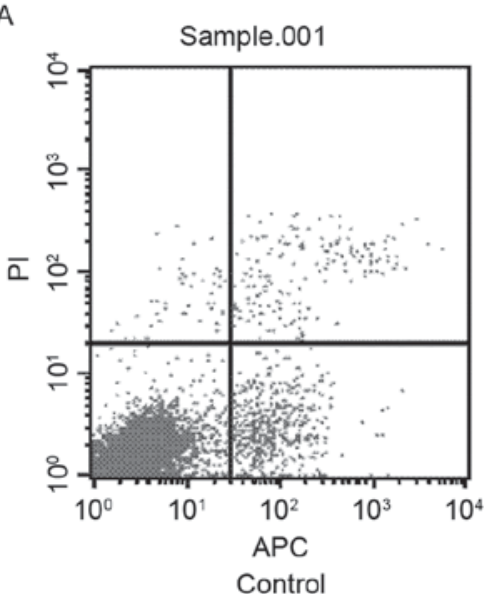

D

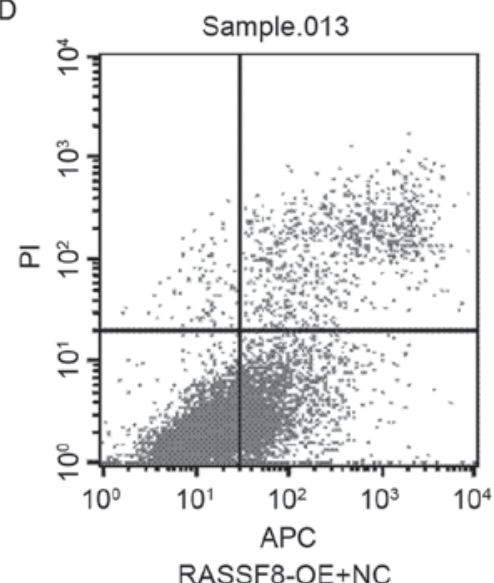

B

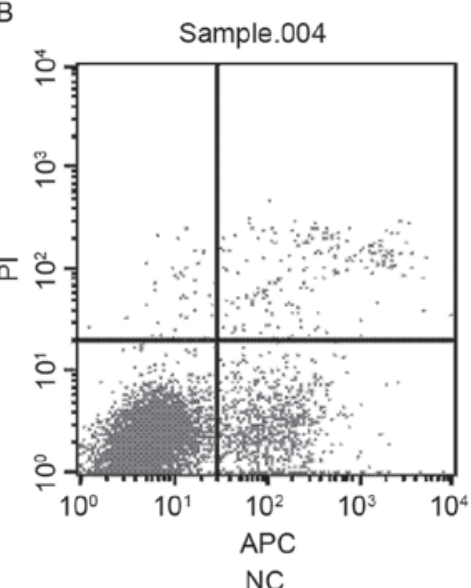

E

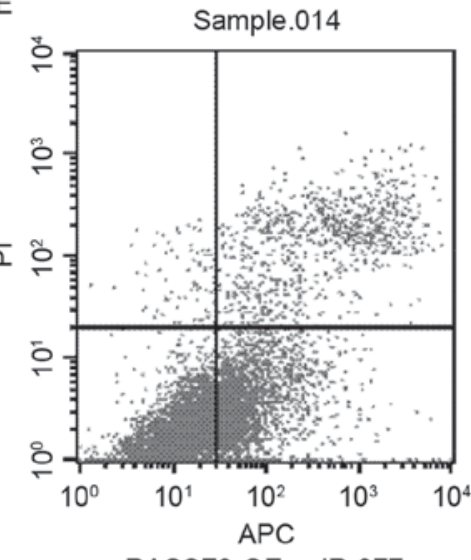

C

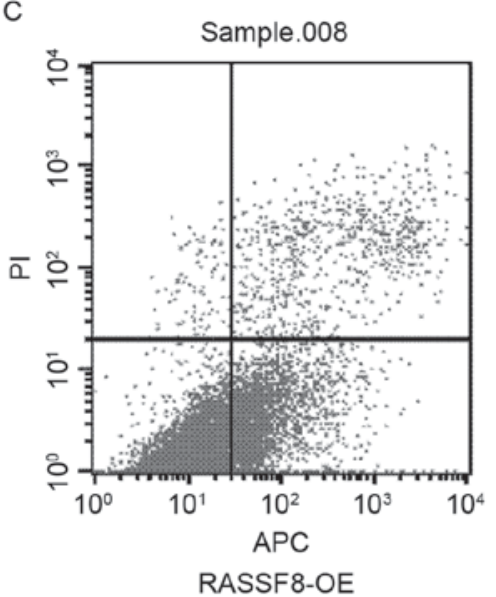

$\mathrm{F}$

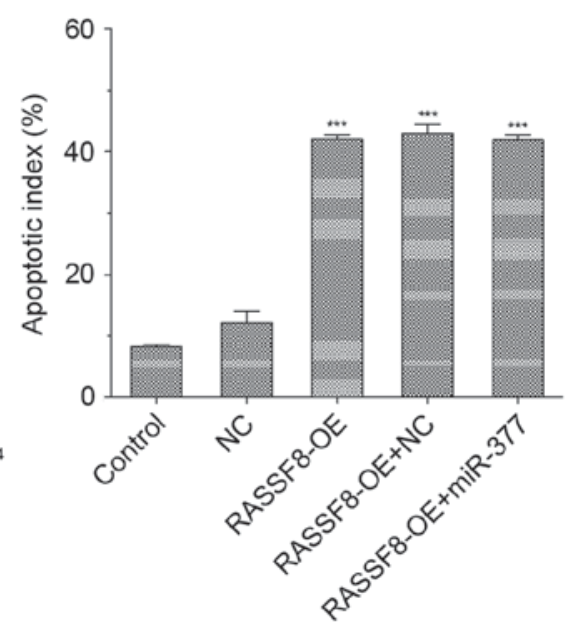

Figure 4. Effect of RASSF8 on apoptosis of BGC-823 cells. Flow cytometric analysis was carried out in BGC-823 cells transfected with control, negative control, RASSF8-OE and RASSF8-OE+ miR-377 at $48 \mathrm{~h}$ after cells were seeded. (A-E) Representative figures of flow cytometry results and rate of apoptosis measured by flow cytometry (F). Data are presented as the mean \pm standard deviation of three independent experiments. ${ }^{* * *} \mathrm{P}<0.001$ vs. control. RASSF8 promotes apoptosis of BGC-823 cells significantly. RASSF8, Ras association domain family 8; miR, microRNA; OE, overexpression; NC, negative control; PI, propidium iodide; APC, allophycocyanin.

Databases for prediction of the targeting relationship. TargetScan (www.targetscan.org/vert_71) and Starbase (starbase.sysu.edu.cn) were used to predict the targeting association between miR-377 and RASSF8.

Luciferase reporter assays. A total of $1 \times 10^{5}$ HEK 293 $\mathrm{T}$ cells/well were seeded into 24 -well culture plates in RPMI-1640 containing 10\% FBS without antibiotics. After $24 \mathrm{~h}$, the cells were cotransfected with miR-NC, miR-377 mimics and the 3'-UTR of RASSF8 responsive firefly luciferase reporter plasmid and Renilla luciferase plasmid (R\&S Biotechnology Co., Ltd., Shanghai, China) and transfection reagent (POLO deliverer TM 3000 Transfection Reagent POLO3000, CT001; R\&S Biotechnology Co., Ltd.) according to the manufacturer's protocol. Cells were incubated for $24 \mathrm{~h}$ under normal conditions, then cell lysates were prepared and luciferase activities were measured using the Dual-Luciferase Reporter Assay system (Promega Corporation). Firefly luciferase activity was normalized to the activity of Renilla luciferase.

Statistical analysis. Results are presented as the means \pm standard deviation of three independent samples. A comparison of the level of RASSF/miR-377 expression between gastric cancer and adjacent normal tissue was performed using the Wilcoxon signed-rank test. Significant differences in the mean values were evaluated using the Student's unpaired t-test. Where multiple comparisons were required, analysis was performed using one-way analysis of variance with Bonferroni correction. $\mathrm{P}<0.05$ was considered to indicate a statistically significant difference.

\section{Results}

Expression of RASSF8 and miR-377 in human gastric cancer tissues and cell lines. The overall expression levels of RASSF8 and miR-377 human gastric cancer tissues, and cell lines were determined using RT-qPCR. As presented in Fig. 1A, levels of RASSF8 in the human GES-1 normal cells were significantly higher compared with the levels of RASSF8 in BGC-823, AGS, MKN-45, HGC-27 and SGC-7901. In addition, RASSF8 expression in tumor tissues demonstrated significantly attenuated levels compared with the corresponding normal tissues, with a mean1.6-fold decrease (Fig. 1B). Furthermore, levels of miR-377 in the 
A
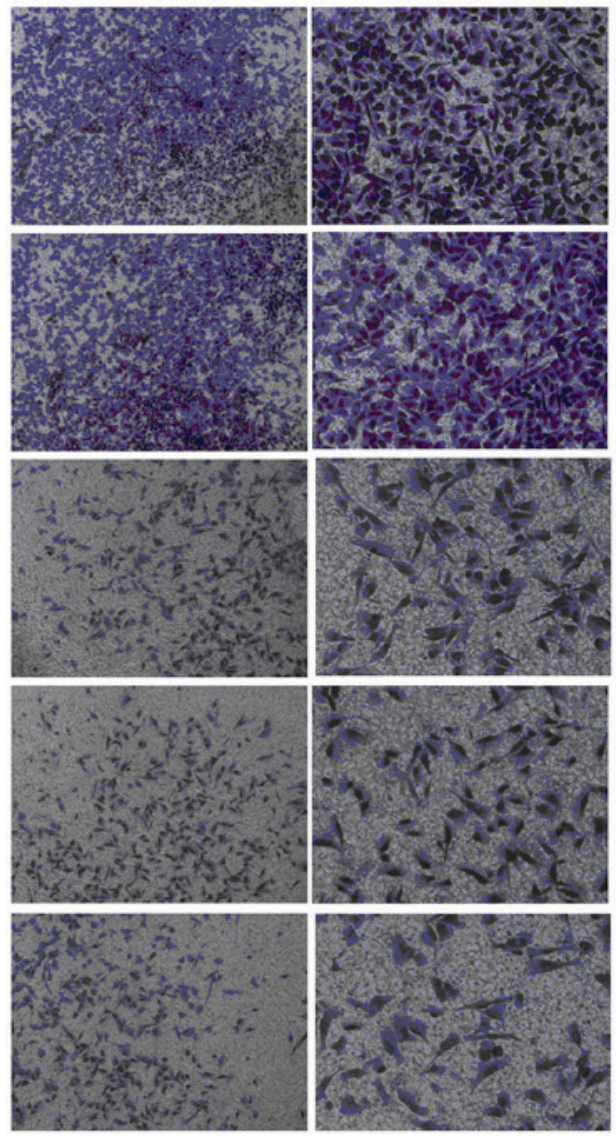

Control

RASSF8-NC

RASSF8-OE

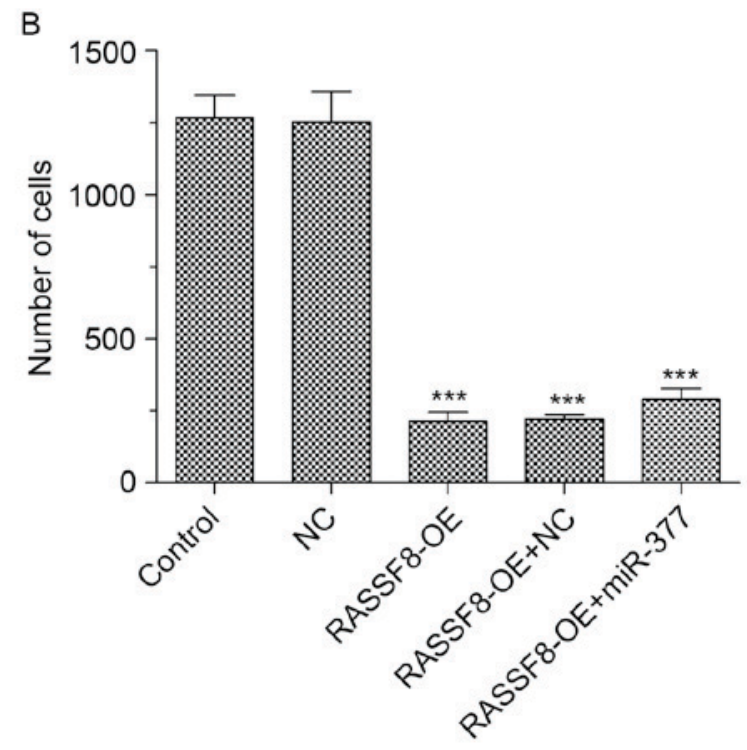

RASSF8-OE

+ miR-377-NC

Figure 5. RASSF8 inhibits cell invasion in BGC-823 cells. (A) Transwell invasion assay in BGC-823 cells transfected with control, negative control, RASSF8-OE and RASSF8-OE+ miR-377 at $24 \mathrm{~h}$ after cells were seeded. (B) The invaded cell numbers are quantified using ImageJ software. Data are presented as the mean \pm standard deviation of three independent experiments. ${ }^{* * * *} \mathrm{P}<0.001$ vs. control. RASSF8, Ras association domain family 8 ; miR, microRNA; OE, overexpression; NC, negative control.

BGC-823, AGS and MKN-45 cell lines were significantly higher compared with that in the normal GES-1 cell line $(\mathrm{P}<0.01$; Fig. 1C), which was consistent with a previous report (24). However, no significant differences were identified among the levels of miR-377 in the HGC-27, SGC-7901 and normal GES-1 cell lines in the present study. Furthermore, a significantly increased expression level of miR-377 between the tumor and normal groups was identified, with a mean 20.4-fold increase $(\mathrm{P}<0.05$; Fig. 1D).

RASSF8 was immunohistochemically stained in the tissue sections of gastric cancer and their corresponding adjacent non-cancerous mucosa. It was demonstrated that the RASSF8 protein was abundantly expressed in the upper glandular layer of the superficial epithelium, while expression of RASSF8 protein was significantly downregulated in gastric cancer tissue compared with normal gastric mucosa (Table I; Fig. 2; $\mathrm{P}<0.05)$.

RASSF8 attenuates gastric cancer cell proliferation. To examine the role of RASSF8/miR-377 in tumorigenesis of gastric cancer, the effect of RASSF8 overexpression and miR-377 on the proliferation of BGC-823 gastric cancer cell lines was examined. Following infection with RASSF8 overexpression lentivirus the cells were transfected with miR-377 mimic or the miR377-NC. The RT-qPCR results demonstrated that RASSF8 was significantly increased in the cells transfected with RASSF8 overexpression lentivirus (Fig. 3A). The CCK-8 assay demonstrated that the overexpression of RASSF8 significantly suppressed the proliferation of BGC-823 cells, whereas miR-377 attenuated the effect of RASSF8 on cell proliferation (Fig. 3B). The proliferation of the RASSF8 overexpression with miR377 group remained significantly lower compared with that of the negative control group, in which cells were transfected by negative control miRNA. The colony formation assay was performed to further confirm the effect of RASSF8/miR-377 on gastric cancer cell proliferation, and the data indicated that the overexpression of RASSF8 significantly decreased colony numbers in BGC-823 cell cultures, which was not reversed by treatment with miR-377, which confirmed the results of CCK-8 assay (Fig. 3C). Furthermore, the cell cycle distribution was assessed by flow cytometry. As presented in Fig. 3D, the overexpression ofRASSF8decreased the proportion of cells in the $\mathrm{G}_{1}$ phase at 24,48 and $72 \mathrm{~h}$, compared with the control (BGC-823 cells without transfection) or negative control. Additionally, overexpression of RASSF8 increased the proportion of cells in the $\mathrm{S}$ phase at 24 and $48 \mathrm{~h}$, compared with the control or negative control, and at $72 \mathrm{~h}$, compared with the control, as well slightly decreased at $72 \mathrm{~h}$ compared with the negative control and led to cell cycle arrests in BGC-823 cells. Similarly, miR-377 attenuated rather than reversed the effect of 
RASSF8 on cell cycle. Collectively, these results demonstrated that RASSF8 inhibits gastric cancer cell growth.

RASSF8 promotes gastric cancer cell apoptosis. As presented in Fig. 4, RASSF8 overexpression significantly increased the rate of apoptotic cells compared with the control $(\mathrm{P}<0.01)$. Furthermore, no significant differences were identified between the RASSF8 and RASSF8 combined with miR-377 groups regarding the rate of apoptotic cells.

RASSF8 inhibits the invasion capability of BGC-823 cells. To investigate the effect of RASSF8 ontheinvasivecapability of gastric cancer cells, Transwell assays were performed in BGC-823 cells. Fig. 5A demonstrates the changes in theinvasive capacity of BGC-823 cells. BGC-823 cells infected with RASSF8 overexpression lentivirus revealed significantly decreased invasive capabilities compared with NC lentivirus infected-BGC-823 cells, with an $80 \%$ reduced ability to invade through Matrigel membranes (Fig. 5B). Compared with the RASSF8 overexpression group, no significant differences were observed in the invasive capabilities of BGC-823 cells transfected with RASSF8 and miR-377. Collectively, these results suggest that RASSF8 decreased BGC-823 cell invasion capability and miR-377 could not reverse the effect of RASSF8 on BGC-823 cell invasion.

miR-377 directly targets RASSF8. To gain insight into the biological implications of RASSF8/miR-377 on gastric cancer tumorigenesis, TargetScan, miRanda and DIANA were used to identify putative human protein-coding gene targets of miR-377. The tumor suppressor gene RASSF8 was predicted to have miR-377-binding elements in its 3'-UTRs with high fidelity scores (Fig. 6A). To assess whether the predicted miR-377-binding sites in the 3'-UTR of the target gene was responsible for miR-377 regulation, the 3'-UTR regions downstream of a luciferase reporter gene were cloned and transfected into the BGC-823 control, RASSF8 overexpression with miR-377, and RASSF8 overexpression with miR-377-NC groups. The luciferase activity of RASSF8-overexpressed cells was significantly increased compared with the control. miR-377 significantly suppressed the luciferase activity of RASSF8-overexpressed cells, which was not observed following transfection with miR-377-NC (Fig. 6B). However, miR-377 attenuated but not reversed the luciferase activity of cells transfected with RASSF8 overexpression compared with control, which suggested that miR-377 could decreased but not inhibit the effect of overexpression of RASSF8 compared with control or negative control.

\section{Discussion}

In the current study, the expression of RASSF8/miR-377 was investigated in gastric cancer cell lines and tissue specimens. RASSF8 expression was identified to be significantly upregulated in normal gastric mucosa adjacent tissue compared with that in gastric cancer tissue, indicating that the loss of RASSF8 expression may contribute to gastric carcinogenesis. The current immunohistochemical data also revealed that RASSF8 protein was absent in the gastric cancer tissue and expressed in normal adjacent gastric mucosa. However,
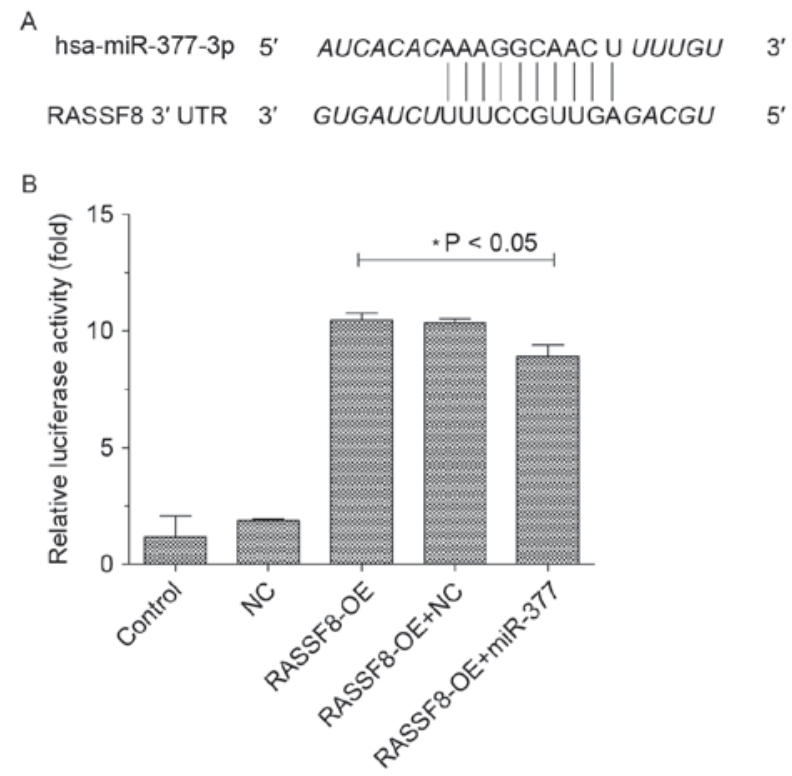

C

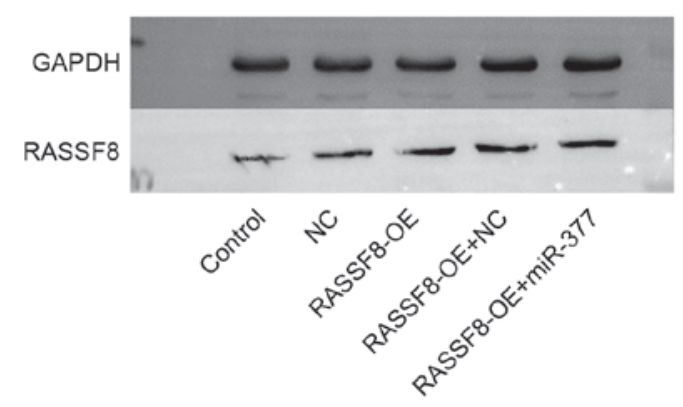

Figure 6. RASSF8 is direct target of miR377. (A) Sequences of the putative miR-377 binding sites in the 3'-UTR of RASSF8. (B) Luciferase assay of BGC-823 cells transfected with hRluc-RASSF8-3'UTR and control, negative control, RASSF8-OE, RASSF8-OE+ miR-377, respectively. (C) Western blot analysis of RASSF8 in BGC-823 cells transfected with control, negative control, RASSF8-OE or RASSF8-OE+ miR-377. Data are presented as the mean \pm standard deviation of three independent experiments. RASSF8, Ras association domain family 8; miR, microRNA; OE, overexpression; NC, negative control; UTR, untranslated region.

miR-377 was significantly upregulated in gastric cancer tissues compared with in their normal adjacent mucosa, indicating that the upregulation of miR-377 expression may contribute to gastric carcinogenesis, which was consistent with a previous report (24).

To further investigate the significance of RASSF8/miR-377 in gastric cancer, RASSF8 was successfully cloned and transfected into gastric cancer BGC-823 cells that overexpressed RASSF8 protein, and investigated the effect of miR377 on RASSF8. Cell growth assays on BGC-823 cells were performed to assess the effect of differential RASSF8/miR-377 expression on cell viability. The data of the colony formation assay in BGC-823 cells further confirmed the effect of RASSF $8 / m i R 377$ on cell growth. These results indicated that the upregulation of RASSF8 expression significantly suppressed cell growth. The effect of RASSF8 on cancer cell growth was attenuated, but not reversed by miR-377. When assessing the cell cycle of BGC-823 cells by flow cytometry, it was identified that RASSF8 overexpression resulted in a population with more cells in the $\mathrm{S} / \mathrm{G}_{2} / \mathrm{M}$-phase, and fewer 
cells in the $\mathrm{G}_{1}$-phase. However, cells transfected with RASSF8 overexpression lentivirus and miR-377-mimic yielded a population with more cells in the S-phase and fewer cells in $\mathrm{G}_{2} / \mathrm{M}$-phase compared with RASSF8 overexpression cells. The following apoptosis data demonstrated that RASSF8 may induce apoptosis in gastric cancer cells while miR-377 may inhibit apoptosis. The results of the present study indicated that RASSF8 may suppress gastric cancer cell proliferation by arresting cells in the $\mathrm{S} / \mathrm{G}_{2} / \mathrm{M}$-phase, whereas miR-377 increased the percentage of cells in the S-phase, thus promoting gastric cancer cell growth. Furthermore, miR-377 significantly increased the percentage of cells in the $\mathrm{G}_{2} / \mathrm{M}$-phase and decreased that in the S-phase correspondingly at $72 \mathrm{~h}$, which confirmed the effect and mechanism of miR377 in gastric cancer cells proliferation. The data of the Transwell assay in BGC-823 cells suggested that RASSF8 significantly inhibited BGC-823 cell invasion capability, which could not be reversed bymiR377. As previously reported, RASSF8 is essential for maintaining adherent junction function, and is involved in regulating the migration of epithelial cells and inhibiting cell proliferation $(25,23)$, the results of the present study suggested that RASSF8 can inhibit BGC-823 cell invasion capability and suppress gastric cancer cell proliferation.

To investigate the association between RASSF8 and miR-377 in gastric cancer, a luciferase-reporter system was developed to confirm whether miR-377 can regulate RASSF8. The results suggested that miR-377 could target to RASSF8. In previous reports, miR-377 was identified to be overexpressed in tumor tissue and cell lines, in addition to being associated with tumorigenesis, and poor prognosis $(3,13)$, which is consistent with the observations of the present study whereby BGC-823 cells exhibit increased miR-377 expression. Furthermore, miR-377 has been identified to increase the proliferation ability and cell invasion capability and decrease the apoptosis of gastric cancer cells (24). Notably, in the present studymiR-377 was not able to reverse the effect of RASSF8 on proliferation, apoptosis and cell invasion of BGC-823 cells.

In conclusion, the results of present study demonstrated that RASSF8 was overexpressed in normal adjacent gastric mucosa compared with gastric cancer tissue. However, miR-377 was overexpressed in gastric cancer tissue and cell lines. RASSF8 overexpression inhibited proliferation ability, apoptosis and invasion capability of BGC-823 cells. It was suggested that miR-377 directly targets RASSF8 through binding to its 3'-UTR, thus increasing the cell proliferative and invasive capabilities, while decreasing the apoptosis of gastric cancer cells. miR-377 attenuated, but did not reverse the effect of RASSF8 on proliferation, apoptosis and cell invasion in BGC-823 cells. Thus, the RASSF8 gene may represent a novel molecular target involved in gastric cancer development and may offer a promising therapeutic approach for the treatment of patients with gastric cancer.

\section{References}

1. Gibson CJ, Britton KA, Miller AL and Loscalzo J: Clinical problem-solving. Out of the blue. N Engl J Med 370: 1742-1748, 2014.

2. Wu WK, Lee CW, Cho CH, Fan D, Wu K, Yu J and Sung JJ: MicroRNA dysregulation in gastric cancer: A new player enters the game. Oncogene 29: 5761-5771, 2010.
3. Catalano V, Labianca R, Beretta GD, Gatta G, de Braud F and Van Cutsem E: Gastric cancer. Crit Rev Oncol Hematol 71: 127-164, 2009.

4. Gomceli I, Demiriz B and Tez M: Gastric carcinogenesis. World J Gastroenterol 18: 5164-5170, 2012.

5. Beckman JD, Chen C, Nguyen J, Thayanithy V, Subramanian S, Steer CJ and Vercellotti GM: Regulation of heme oxygenase-1 protein expression by miR-377 in combination with miR-217. J Biol Chem 286: 3194-3202, 2011

6. Mascaux C, Iannino N, Martin B, Paesmans M, Berghmans T, Dusart M, Haller A, Lothaire P, Meert AP, Noel S, et al: The role of RAS oncogene in survival of patients with lung cancer: A systematic review of the literature with meta-analysis. Br J Cancer 92: 131-139, 2005.

7. Ellis CA and Clark G: The importance of being K-Ras. Cell Signal 12: 425-434, 2000.

8. Falvella FS, Manenti G, Spinola M, Pignatiello C, Conti B, Pastorino U and Dragani TA: Identification of RASSF8 as a candidate lung tumor suppressor gene. Oncogene 25: 3934-3938, 2006.

9. Malumbres M and Barbacid M: RAS oncogenes: The first 30 years. Nat Rev Cancer 3: 459-465, 2003.

10. Downward J: Targeting RAS signalling pathways in cancer therapy. Nat Rev Cancer 3: 11-22, 2003.

11. Wohlgemuth S, Kiel C, Krämer A, Serrano L, Wittinghofer F and Herrmann C: Recognizing and defining true Ras binding domains I: Biochemical analysis. J Mol Biol 348: 741-758, 2005.

12. Sherwood V, Manbodh R, Sheppard C and Chalmers AD: RASSF7 is a member of a new family of RAS association domain-containing proteins and is required for completing mitosis. Mol Biol Cell 19: 1772-1782, 2008.

13. Zehavi L, Schayek H, Jacob-Hirsch J, Sidi Y, Leibowitz-Amit R and Avni D: MiR-377 targets E2F3 and alters the NF-kB signaling pathway through MAP3K 7 in malignant melanoma. Mol Cancer 14: $68,2015$.

14. Ueda T, Volinia S, Okumura H, Shimizu M, Taccioli C, Rossi S, Alder H, Liu CG, Oue N, Yasui W, et al: Relation between microRNA expression and progression and prognosis of gastric cancer: A microRNA expression analysis. Lancet Oncol 11: 136-146, 2010.

15. Rachagani S, Kumar S and Batra SK: MicroRNA in pancreatic cancer: Pathological, diagnostic and therapeutic implications. Cancer Lett 292: 8-16, 2010.

16. Wang QZ, Xu W, Habib N and Xu R: Potential uses of microRNA in lung cancer diagnosis, prognosis, and therapy. Curr Cancer Drug Targets 9: 572-594, 2009.

17. Balch C,Fang F,Matei DE, Huang TH and Nephew KP: Minireview: Epigenetic changes in ovarian cancer. Endocrinology 150: 4003-4011, 2009.

18. Faber C, Kirchner T and Hlubek F: The impact of microRNAs on colorectal cancer. Virchows Arch 454: 359-367, 2009.

19. Mott JL: MicroRNAs involved in tumor suppressor and oncogene pathways: Implications for hepatobiliary neoplasia. Hepatology 50: 630-637, 2009.

20. Inui M, Martello G and Piccolo S: MicroRNA control of signal transduction. Nat Rev Mol Cell Biol 11: 252-263, 2010.

21. Wu C, Guo X, Wang W, Wang Y, Shan Y, Zhang B, Song W, Ma S, Ge J, Deng H and Zhu M: N-Acetylgalactosaminyltransferase-14 as a potential biomarker for breast cancer by immunohistochemistry. BMC Cancer 10: 123, 2010.

22. Livak KJ and Schmittgen TD: Analysis of relative gene expression data using real-time quantitative PCR and the 2(-Delta Delta C(T)) method. Methods 25: 402-408, 2001.

23. Lang Y, Xu S, Ma J, Wu J, Jin S, Cao S and Yu Y: MicroRNA-429 induces tumorigenesis of human non-small cell lung cancer cells and targets multiple tumor suppressor genes. Biochem Biophys Res Commun 450: 154-159, 2014.

24. Wen X, Wu JQ, Peng W, Feng JF and Tang JH: MicroRNA-377 predicts poor clinical outcome of gastric cancer and induces tumorigenesis by targeting multiple tumor-suppressor genes. Oncol Rep 34: 203-210, 2015.

25. Lock FE, Underhill-Day N, Dunwell T, Matallanas D, Cooper W, Hesson L, Recino A, Ward A, Pavlova T, Zabarovsky E, et al: The RASSF8 candidate tumor suppressor inhibits cell growth and regulates the Wnt and NF-kappaB signaling pathways. Oncogene 29: 4307-4316, 2010.

This work is licensed under a Creative Commons Attribution-NonCommercial-NoDerivatives 4.0 International (CC BY-NC-ND 4.0) License. 